\title{
The Distinguishing Chromatic Number
}

\author{
Karen L. Collins \\ Dept. of Math. and Comp. Sci. \\ Wesleyan University \\ Middletown, CT 06459-0128 \\ kcollins@wesleyan.edu
}

\author{
Ann N. Trenk \\ Dept. of Math. \\ Wellesley College \\ Wellesley, MA 02481 \\ atrenk@wellesley.edu
}

Submitted: Jun 10, 2005; Accepted: Nov 6, 2005; Published: Feb 15, 2006

Mathematics Subject Classification: 05C15, 05C25

\begin{abstract}
In this paper we define and study the distinguishing chromatic number, $\chi_{D}(G)$, of a graph $G$, building on the work of Albertson and Collins who studied the distinguishing number. We find $\chi_{D}(G)$ for various families of graphs and characterize those graphs with $\chi_{D}(G)=|V(G)|$, and those trees with the maximum chromatic distingushing number for trees. We prove analogs of Brooks' Theorem for both the distinguishing number and the distinguishing chromatic number, and for both trees and connected graphs. We conclude with some conjectures.
\end{abstract}

\section{Introduction}

In [1], Albertson and Collins study the distinguishing number of a graph, inspired by the following problem: given a ring of seemingly identical keys that open different doors, how many colors are needed to distinguish them? In the language of graph theory, this is the question of how many colors are needed to color the vertices of the cycle $C_{n}$ so that the only automorphism of the graph which preserves colors is the identity. In this problem there is no requirement that the coloring be proper. Interestingly, the cycles $C_{3}$, $C_{4}$, and $C_{5}$ require 3 colors, but cycles with 6 or more vertices need only 2 colors (see Theorem 2.2). Albertson and Collins study the problem for graphs in general using the following definitions.

Definition 1.1 A labeling of the vertices of a graph $\mathrm{G}, h: V(G) \rightarrow\{1, \ldots, r\}$, is said to be $r$-distinguishing (or just distinguishing) provided no automorphism of the graph preserves all of the vertex labels. The distinguishing number of a graph $\mathrm{G}$, denoted by $D(G)$, is the minimum $r$ such that $G$ has an $r$-distinguishing labeling. 
In this paper, we study the problem of finding $r$-distinguishing labelings which are also proper colorings.

Definition 1.2 A labeling of the vertices of a graph $\mathrm{G}, h: V(G) \rightarrow\{1, \ldots, r\}$, is said to be proper $r$-distinguishing (or just proper distinguishing) if it is a proper labeling (i.e., coloring) of the graph and no automorphism of the graph preserves all of the vertex labels. The distinguishing chromatic number of a graph $G, \chi_{D}(G)$, is the minimum $r$ such that $G$ has a proper $r$-distinguishing labeling.

This definition is related to the following problem of assigning rooms to a set of meetings. Represent the set of meetings using a graph where there is a vertex for each meeting, and two vertices are adjacent precisely when the meetings conflict, i.e., overlap in time. Assigning colors to the vertices of this graph would assign rooms to the meetings, and a proper coloring would ensure that meetings that conflict would be assigned to different rooms. The chromatic number of this graph is the minimum number of rooms necessary to accommodate all the meetings. If the coloring was done so that it was not only a proper coloring, but also a distinguishing coloring, then the graph and its coloring would uniquely identify the meetings as well as specifying which room each would use.

We make analogous definitions for rooted graphs. A rooted graph $(G, z)$ is a graph $G$ together with a vertex $z \in V(G)$ designated as the root. A labeling of the vertices of a rooted graph $\mathrm{G}, h: V(G) \rightarrow\{1, \ldots, r\}$, is said to be $r$-distinguishing provided no automorphism of the graph preserves all of the vertex labels and fixes the root $z$. The distinguishing chromatic number of a rooted graph $(G, z), \chi_{D}(G, z)$, is the minimum $r$ such that $(G, z)$ has a proper $r$-coloring that is also $r$-distinguishing.

\section{Preliminary Results}

In this section we present a table of results that shows the chromatic number, the distinguishing number and the distinguishing chromatic number for $\overline{K_{n}}$ and various families of connected graphs. In addition, we characterize those graphs $G$ for which the only way to achieve a proper $r$-distinguishing labeling is to use a different color for each vertex, i.e., $\chi_{D}(G)=|V(G)|$. We begin with a few observations.

Observations 2.1 1. For any graph $G, \chi_{D}(G) \geq \max \{\chi(G), D(G)\}$.

2. If a graph $G$ has no non-trivial automorphisms, then $\chi_{D}(G)=\chi(G)$ and $D(G)=1$. Hence $\chi_{D}(G)$ can be much larger than $D(G)$.

3. Let $G \vee H$ be the join of graphs $G$ and $H$, which consists of a copy of $G$, a copy of $H$ and all possible edges between them. Then $\chi_{D}(G \vee H)=\chi_{D}(G)+\chi_{D}(H)$, just as $\chi(G \vee H)=\chi(G)+\chi(H)$.

Theorem 2.2 The entries in Table 1 are correct. 


\begin{tabular}{|l|l|c|c|c|}
\hline & Graph $G$ & $\chi(G)$ & $D(G)$ & $\chi_{D}(G)$ \\
\hline 1. & $K_{n}, \Delta=n-1$ & $n$ & $n$ & $n$ \\
\hline 2. & Complement of $K_{n}$ & 1 & $n$ & $n$ \\
\hline 3. & $K_{a_{1} j_{1}, a_{2} j_{2}, \ldots, a_{r} j_{r}}$ & $\sum j_{i}$ & Thm 2.4 & $\sum j_{i} a_{i}$, Thm 2.3 \\
\hline \hline 4. & $P_{2 n}, \Delta \leq 2$ & 2 & 2 & 2 \\
\hline 5. & $P_{2 n+1}, \Delta \leq 2$ & 2 & 2 & 3 \\
\hline \hline 6. & $C_{4}, \Delta=2$ & 2 & 3 & 4 \\
\hline 7. & $C_{5}, \Delta=2$ & 3 & 3 & 3 \\
\hline 8. & $C_{6}, \Delta=2$ & 2 & 2 & 3 \\
\hline 9. & $C_{2 n}, n \geq 4, \Delta=2$ & 2 & 2 & 3 \\
\hline 10. & $C_{2 n+1}, n \geq 3, \Delta=2$ & 3 & 2 & 4 \\
\hline \hline 11. & Petersen graph, $\Delta=3$ & 3 & 3 & $\Delta, 1$, Thm 3.4 \\
\hline 12. & $T_{\Delta, h}, \Delta \geq 2$ & 2 & $\Delta$, Thm 3.5 & $\Delta+1$ Thm 3.4 \\
\hline 13. & Tree $T \neq T_{\Delta, h}, K_{1}, K_{2}$ & 2 & $\leq \Delta-1$ Thm 3.5 & $\leq \Delta$, Thm 4.5 \\
\hline 14. & $G$ connected & $\leq \Delta+1[2]$ & $\leq \Delta+1$, Thm 4.2 & $\leq 2 \Delta$, Thm \\
\hline
\end{tabular}

Table 1: Table of results for $\chi, D$ and $\chi_{D}$

Proof The chromatic numbers for the classes of graphs given in this table are well-known, we justify the entries in the columns labeled $D(G)$ and $\chi_{D}(G)$.

Complete multipartite graphs (rows 1-3): The distinguishing chromatic number of a complete multipartite graph is determined by Theorem 2.3 and the distinguishing number of a complete multipartite graph is determined by Theorem 2.4. Hence, the example of complete bipartite graphs show that $\chi_{D}(G)$ can be much larger than $\chi(G)$.

Paths (rows 4-5): The path $P_{m}$ has a non-trivial automorphism for all $m \geq 2$, thus $D\left(P_{m}\right) \geq 2$. For even values of $m$, any proper 2-coloring of $P_{m}$ is also distinguishing, thus $\chi_{D}\left(P_{2 m}\right)=D\left(P_{2 m}\right)=2$. For odd values of $m$, the labeling that uses color 2 for one end-vertex and color 1 for the remaining vertices is distinguishing, thus $D\left(P_{2 n+1}\right)=$ 2. However, it is not a proper coloring and any proper coloring using two colors is not distinguishing, so $\chi_{D}\left(P_{2 n+1}\right) \geq 3$. A 3-labeling that is proper and distinguishing is achieved by using 1 for an end-vertex and alternating 2's and 3's for the remaining vertices, thus $\chi_{D}\left(P_{2 n+1}\right) \leq 3$. The results for the distinguishing chromatic number of paths can also be obtained as special cases of Theorem 3.4 where $P_{2 n+1}=T_{2, n}$ and $P_{2 n}$ is a tree which is not of the form $T_{\Delta, h}$.

Cycles (rows 6-10): The cycle $C_{4}$ is the complete multipartite graph $K_{2,2}=K_{2^{2}}$ which has $D\left(C_{4}\right)=3$ and $\chi_{D}\left(C_{4}\right)=4$ as discussed above. The distinguishing numbers of cycles are computed in [1] as follows. The cycle $C_{5}$ has $D\left(C_{5}\right)=3$ and the cycle $C_{n}$ with vertices labeled consecutively as $v_{0}, v_{1}, \ldots, v_{n-1}$ for $n \geq 6$ has $D\left(C_{n}\right)=2$ using color 1 for vertices $v_{0}, v_{2}$ and $v_{3}$ and color 2 for the remaining vertices.

We next consider the distinguishing chromatic number of cycles $C_{n}$ for $n \geq 5$. It is easy to see that $\chi_{D}\left(C_{5}\right)=3$ and somewhat harder to check that $\chi_{D}\left(C_{6}\right)=4$. For $n \geq 7$, let the vertices be labeled consecutively as $v_{0}, v_{1}, \ldots, v_{n-1}$ and use color 3 for vertices 
$v_{0}$ and $v_{3}$, color 2 for vertices $v_{i}$ where $i \neq 3$ is odd and color 1 for vertices $v_{i}$ where $i \neq 0$ is even. This shows $\chi_{D}\left(C_{n}\right) \leq 3$ for $n \geq 7$. All proper 2-colorings of $C_{n}$ have color-preserving automorphisms, thus $\chi_{D}\left(C_{n}\right)>2$ for all $n$ and in particular, $\chi_{D}\left(C_{n}\right)=3$ for $n \geq 7$.

Misc. (rows 11-14): The tree $T_{k, h}$ is defined in Definition 3.3. The remaining results for $D(G)$ and $\chi_{D}(G)$ in Table 1 are shown in Theorems 2.5, 3.4, 3.5, 4.2, and 4.5.

Theorem 2.3 Let $G$ be a graph. Then $\chi_{D}(G)=|V(G)|$ iff $G$ is a complete multipartite graph.

Proof $(\Longleftarrow)$ Suppose that two vertices of $G$ are given the same color in a proper coloring. Since they are not adjacent, they must be in the same partite set. However, there is an automorphism of $G$ which interchanges those two vertices and leaves the rest of the graph fixed. Alternatively, one can use Observation 2.1.3.

$(\Longrightarrow)$ For a contradiction, assume that $G$ is not a complete multipartite graph, but $\chi_{D}(G)=|V(G)|=n$. Then $G$ must have non-adjacent vertices, otherwise $G=K_{n}$ which is a complete $n$-partite graph. Further, $G$ must have a pair of non-adjacent vertices, $u, v$, with different neighborhoods. Color both $u$ and $v$ using color 1 , and color the remaining vertices with $2,3, \ldots, n-1$, where each gets a distinct color. This is a proper coloring, which is $(n-1)$-distinguishing. So $\chi_{D}(G) \leq n-1$, a contradiction.

Let $K_{a_{1} j_{1}, a_{2} j_{2}, \ldots, a_{r} j_{r}}$ denote the complete multipartite graph that has $j_{i}$ partite sets of size $a_{i}$ for $i=1,2, \ldots, r$ and $a_{1}>a_{2}>\cdots>a_{r}$. In finding the distinguishing number of a complete multipartite graph, we need enough colors to distinguish between vertices in the same partite set as well as distinguishing between partite sets of the same cardinality.

Theorem 2.4 Let $K_{a_{1} j_{1}, a_{2} j_{2}, \ldots, a_{r} j_{r}}$ denote the complete multipartite graph that has $j_{i}$ partite sets of size $a_{i}$ for $i=1,2, \ldots, r$ and $a_{1}>a_{2}>\cdots>a_{r}$. Then $D\left(K_{a_{1} j_{1}, a_{2} j_{2}, \ldots, a_{r} j_{r}}\right)=$ $\min \left\{p:\left(\begin{array}{c}p \\ a_{i}\end{array}\right) \geq j_{i}\right.$ for all i $\}$.

Proof Let $G=K_{a_{1} j_{1}, a_{2} j_{2}, \ldots, a_{r} j_{r}}$. There is an automorphism of $G$ that interchanges any pair of vertices in the same partite set, thus each vertex in a partite set must get a different color. Furthermore, there must be enough colors to distinguish one partite set from the others of the same size. So the sets of colors that appear on the vertices of each of the $j_{i}$ partite sets of size $a_{i}$ must be distinct, hence $\left(\begin{array}{l}D(G) \\ a_{i}\end{array}\right) \geq j_{i}$ for each $i$. Any such coloring is distinguishing, so $D(G)$ is the smallest integer $p$ for which $\left(\begin{array}{l}p \\ a_{i}\end{array} \geq j_{i}\right.$ for all $i$.

Theorem 2.5 If $P$ is the Petersen graph then $\chi_{D}(P)=4$.

Proof It is shown in [1] that $D(P)=3$. Since $\chi(P)=3$, we need only show that $\chi_{D}(P)>3$ and $\chi_{D}(P) \leq 4$. Recall that the Kneser graph, $K G(r, s)$, is the graph whose vertices are in a $1-1$ correspondence with the $r$-sized subsets of $\{1,2,3, \ldots, s\}$, and there 


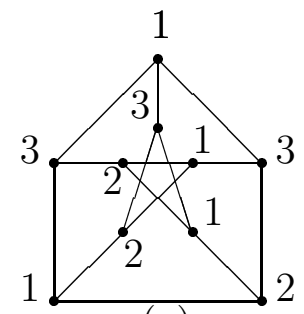

(a)

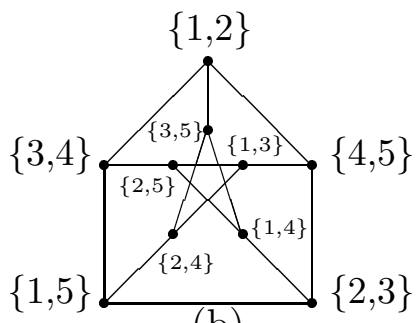

(b)

Figure 1: (a) The Petersen graph, $P$ with a proper 3-coloring, and (b) $K G(2,5)$.

is an edge between two vertices if their corresponding $r$-subsets are disjoint [4]. The Petersen graph is $K G(2,5)$, see Figure 1(b).

By the Erdős-Ko-Rado Theorem [4], a maximum independent set in a Kneser graph is formed by choosing all subsets that contain a fixed element. Let $I_{j}$ be the set of vertices in $P$ whose corresponding 2 -subsets contain $j$. Thus, $P$ has 5 maximum independent sets $I_{j}$, each contains 4 elements, and each pair $I_{j}, I_{k}$ of distinct maximum independent sets has 1 element in common. This means that every 3-coloring of $P$ has color class partition $4,3,3$.

It is well known that the automorphism group of $P$ is $S_{5}$, acting in the obvious way on the 2 -subsets of $\{1,2,3,4,5\}$. The graph $P-I_{j}$ consists of 3 copies of $K_{2}$, and when these 6 vertices are colored properly using 2 colors, one color class always has the form $I_{k}-\{j, k\}$. Thus, the color class partition of a 3 -coloring of the Petersen graph is

$$
\left\{I_{j}, I_{k}-\{j, k\}, P-I_{j} \cup I_{k}\right\}
$$

for some $1 \leq j, k \leq 5$. See, for example, the 3 -coloring of $P$ in Figure 1 (a). We choose a permutation of $\{1,2,3,4,5\}$ which will fix this coloring of $P$. Let $\sigma: P \rightarrow P$ by any non-trivial permutation of $\{l, m, n\} \subset\{1,2,3,4,5\}$ where $\{l, m, n\} \cap\{j, k\}=\emptyset$. Hence $\chi_{D}(P)>3$.

There are several ways to see that $\chi_{D}(P) \leq 4$. One way is to color all vertices in $I_{1}$ with color 1 , then color $\{3,5\},\{4,5\}$ with color $2,\{2,3\},\{2,5\}$ with color 3 , and $\{2,4\},\{3,4\}$ with color 4 , which is a proper coloring since each color class is an independent set. Each $I_{j}$ sees a different multiset of colors, for $1 \leq j \leq 5$, so this is a 4 -distinguishing coloring.

\section{Results for Trees}

In this section we prove two results about the distinguishing chromatic number of trees. We characterize those trees with distinguishing chromatic number equal to two and prove an analog of Brooks' Theorem. In doing so we find the distinguishing number of $T_{k, h}$ (defined in Definition 3.3).

We begin with some terminology and background, following [5]. A rooted tree $(T, z)$ is a tree $T$ with a distinguished vertex $z$, the root. The depth or level of a vertex $v$ is its 
distance from the root, and the height of a rooted tree is the greatest depth in the tree. The parent of $v$ is the vertex immediately following $v$ on the unique path from $v$ to the root. Two vertices are siblings if they have the same parent. The eccentricity of a vertex $v$ in a graph $G$ is the distance from $v$ to a vertex farthest from $v$. The center of $G$ is the subgraph induced by those vertices with minimum eccentricity. It is well-known that the center of a tree is either a single vertex or an edge.

Theorem 3.1 Let $T$ be a tree with at least 2 vertices. Then $\chi_{D}(T)=2$ iff $T$ has no non-trivial automorphisms, or the center of $T$ is an edge $\{x, y\}$ and $T$ has precisely one non-trivial automorphism, and this automorphism interchanges $x$ and $y$.

Proof $(\Longleftarrow)$ Since $T$ has at least two vertices, $\chi_{D}(T) \geq \chi(T)=2$. Our goal is to show $\chi_{D}(T) \leq 2$, that is, there exists a proper coloring of $T$ which is 2-distinguishing. There is only one proper 2-coloring of the tree $T$ (up to reversing all colors). If $T$ has no non-trivial automorphisms then this coloring is 2-distinguishing as desired.

Otherwise, suppose the center of $T$ is an edge $\{x, y\}, T$ has precisely one non-trivial automorphism $\sigma$, and $\sigma(x)=y$ and $\sigma(y)=x$. Since $\{x, y\} \in E(T)$, vertices $x$ and $y$ must get different colors. But then $\sigma$ does not preserve colors and our coloring is 2-distinguishing as desired.

$(\Longrightarrow)$ Now suppose $\chi_{D}(T)=2$.

\section{Case 1: The center of $T$ is a vertex $v$.}

Consider a proper 2-distinguishing labeling of $T$, where, without loss of generality, we may assume vertex $v$ gets color 1 . Since this coloring of $T$ is proper, all vertices which are at an even distance from $v$ are colored 1 , and all vertices which are at an odd distance from $v$ are colored 2. However, any automorphism of $T$ must fix $v$, because it is the center of $T$, and therefore also preserve the parity of distances from $v$, and thus also preserves this 2-coloring. Since $\chi_{D}(T)=2, T$ can have no nontrivial automorphisms.

Case 2: The center of $T$ is an edge $\{x, y\}$.

Let $T=T_{x} \cup\{x, y\} \cup T_{y}$ where $x \in T_{x}, y \in T_{y}$ are the connected components remaining after removing the edge $\{x, y\}$ from $T$. Consider a proper 2-distinguishing labeling of $T$, where, without loss of generality, we may assume vertex $x$ gets color 1 , and $y$ gets color 2. If $T$ has no non-trivial automorphisms, then we are done. Otherwise, let $\sigma$ be a nontrivial automorphism of $T$. Then $\sigma$ must either fix both $x$ and $y$ or interchange them. First, suppose that $\sigma$ fixes both $x$ and $y$. As in Case 1, all vertices in $T_{x}$ which are at an even distance from $x$ are colored 1 , and all vertices in $T_{x}$ which are at an odd distance from $x$ are colored 2, and $\sigma$ must preserve these distances (and hence colors) since $\sigma$ fixes $x$. Similarly, $\sigma$ preserves the colors in $T_{y}$, and thus preserves all colors in $T$. Therefore, since $\chi_{D}(T)=2, \sigma$ must be the identity, which contradicts its choice as a non-trivial automorphism.

Now to prove uniqueness, suppose that there are two distinct automorphisms of $T$, $\sigma, \tau$, that interchange $x$ and $y$. Then the composition $\sigma \circ \tau^{-1}$ fixes $x$ and $y$, and therefore is the identity. Hence $\sigma=\tau$, which is a contradiction. 


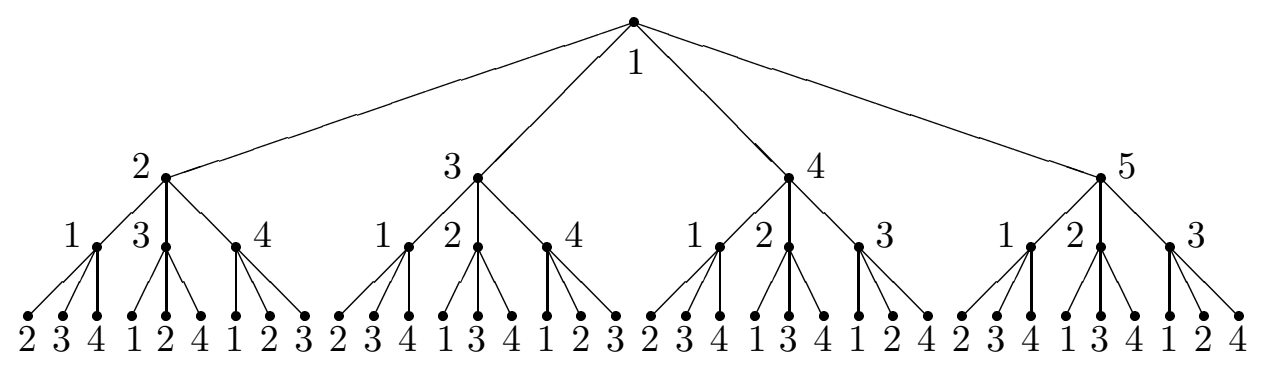

Figure 2: $T_{4,3}$ with a proper 5-distinguishing labeling

Lemma 3.2 A labeling of a rooted tree $(T, z)$ in which each vertex is colored differently from its siblings is a distinguishing labeling. A labeling of a rooted tree $(T, z)$ in which each vertex is colored differently from its siblings and from its parent is a proper distinguishing labeling.

Proof Let $(T, z)$ be a rooted tree whose vertices are colored so that each vertex is colored differently from its siblings (and from its parents). Let $\sigma$ be an automorphism of tree $T$ which preserves colors and fixes the root $z$. We use induction on the level of a vertex to show that all vertices must be fixed by $\sigma$. The only vertex at level 0 is the root $z$, which is fixed. Assume all vertices at level $h$ are fixed by $\sigma$, and let $x$ be a vertex at level $h+1$. By induction, $x$ 's parent (at level $h$ ) is fixed by $\sigma$, thus vertex $x$ must be mapped to itself or one of its siblings. But $x$ is colored differently from its siblings and $\sigma$ preserves colors, hence $x$ is fixed by $\sigma$.

In some situations, the structure of a graph will fix a particular vertex under any automorphism, and in other situations, this will happen only after some of the vertices are assigned colors. We say a vertex $u$ in a graph $G$ in which some vertices have been assigned colors is pinned if $u$ is fixed by every automorphism that preserves colors no matter how the coloring of $G$ is completed. Our goal in finding the distinguishing number or the distinguishing chromatic number is to pin all the vertices, using as few colors as possible.

Definition 3.3 For $k \geq 2$, let $T_{k, h}$ be the rooted tree in which all leaves are at the same distance $h$ from the root, and every vertex that is not a leaf has degree $k$ (including the root).

For example, the tree $T_{4,3}$ is shown in Figure 2 together with a proper 5-distinguishing labeling. Note that $T_{2, h}=P_{2 h+1}$.

A classic result in graph theory is Brooks' Theorem which states that $\chi(G) \leq \Delta(G)+1$ for every connected graph with equality holding only for complete graphs and odd cycles. The following is a distinguishing chromatic number analogue of Brooks' Theorem applied to trees. 
Theorem 3.4 Let $T$ be a tree. Then $\chi_{D}(T) \leq \Delta(T)+1$. Furthermore, equality is achieved iff $T=T_{\Delta, h}$ for some $h \geq 0$.

Proof If $T$ is a single vertex, then the statement of the theorem is obviously true. We next prove that $T$ has a proper $(\Delta+1)$-distinguishing labeling using cases based on whether the center of $T$ is a vertex or an edge.

First, consider the case where the center of $T$ is a vertex $v$ and let $h$ be the height of the rooted tree $(T, v)$. Since $v$ is the center of $T$, all automorphisms of $T$ take $v$ to itself, so $v$ is already pinned. Color $v$ with color 1 . We color the remaining vertices of $T$ using colors from the set $\{1,2,3, \ldots, \Delta+1\}$ as follows. By induction, we may assume that all vertices whose distance to vertex $v$ is less than or equal to $i-1$ are colored properly and pinned, for $1 \leq i \leq h$. Consider the vertices at distance $i$ from $v$ in some fixed order and color each greedily so that each vertex gets the lowest color which has not appeared on its parent or siblings. This is possible because there are $\Delta+1$ colors available to distinctly color a parent and at most $\Delta$ siblings. We will never use color $\Delta+1$ except possibly for one vertex on level 1. Since each vertex is assigned a color different from its parent, this is a proper coloring. By Lemma 3.2, this is a $(\Delta+1)$-distinguishing coloring.

Second, consider the case where the center of $T$ is an edge, $\{x, y\}$. Any automorphism of $T$ must either fix both $x$ and $y$, or interchange them. Color $x$ with 1 and $y$ with 2 . This pins $x$ and $y$. Remove the edge between $x$ and $y$, and consider $x$ as the root of its subtree and $y$ as the root of its subtree, and proceed to color each subtree as in the preceding case. Now we will never use color $\Delta+1$, since $x$ (respectively $y$ ) has at most $\Delta-1$ children in its subtree. Again by Lemma 3.2, this is a proper $\Delta$-distinguishing coloring.

This completes the proof that $\chi_{D}(T) \leq \Delta+1$. In the remainder of the proof, we justify the last sentence of the theorem. First we show that $\chi_{D}\left(T_{\Delta, h}\right)=\Delta+1$ for any $h$. For a contradiction, suppose there is a distinguishing coloring of $T_{\Delta, h}$ using colors $\{1,2,3, \ldots, \Delta\}$. The root $v$ of $T_{\Delta, h}$ has $\Delta$ children, therefore some color must be used at least twice on the children of $v$. Let $u, w$ be two siblings at the greatest depth $d$ which are colored the same. We construct a non-trivial involution $\sigma$ of $T_{\Delta, h}$ which preserves the coloring. Let $\sigma$ fix all vertices which are not in the subtree $U$ rooted at $u$ or the subtree $W$ rooted at $w$. Also, let $\sigma$ interchange $u$ and $w$. By induction on the height assume we have defined the action of $\sigma$ on the vertices in $U \cup W$ at level $d+i, 0 \leq i \leq h-d$. Let $p, q$ be vertices at level $d+i$ such that $\sigma(p)=q$ and $\sigma(q)=p$. Note that this means that $p$ and $q$ have the same color, say color 1. By definition, the children of $p$ have distinct colors, namely, $\{2,3,4, \ldots, \Delta\}$ and the same is true for $q$. Map the children of $p$ to the children of $q$ by matching colors. This process can be repeated for each pair of vertices at level $d+i$ interchanged by $\sigma$ to define the action of $\sigma$ on vertices at level $d+i+1$. Thus, $\sigma$ is a non-trivial automorphism which preserves colors, a contradiction. Hence $\chi_{D}\left(T_{\Delta, h}\right)>\Delta$. Since the above proof shows that $\chi_{D}\left(T_{\Delta, h}\right) \leq \Delta+1$, it must be that $\chi_{D}\left(T_{\Delta, h}\right)=\Delta+1$.

Finally, we assume that $T$ is a tree with $\chi_{D}(T)=\Delta+1$ and prove $T=T_{\Delta, h}$ for some $h$. From our proof above, the center of $T$ must be a single vertex $v$ of degree $\Delta$. We will show $T=T_{\Delta, h}$ where $h$ is the height of the rooted tree $(T, v)$. If some two children of $v$ have two different subtrees, then we can color these two children with the same color and 
proceed as in the first case above to get $\chi_{D}(T) \leq \Delta$, a contradiction. Thus, all children of $v$ have identical subtrees. If these identical subtrees are empty, then $T=T_{\Delta, 1}$.

For a contradiction, assume $T \neq T_{\Delta, h}$. Then there must exist a smallest $l<h$ and a vertex $x$ at level $l$ with fewer than $\Delta-1$ children. We know $l \geq 1$.

Since the subtrees rooted at the vertices of level 1 are identical, each level 1 vertex has some descendant at level $l$ which has fewer than $\Delta-1$ children. Let $u$ be the level 1 vertex on the unique path from $x$ to $v$ and let $w$ be any other level 1 vertex. Assign color 1 to vertex $v$ at level 0 , and color 2 to vertices $u, w$. Color the remaining $\Delta-2$ level 1 vertices distinctly from $\{3,4,5, \ldots, \Delta\}$, and color their subtrees properly in a way that pins every vertex, using only $\Delta$ colors, as we have previously shown can be done. We will color the subtrees $U$, rooted at $u$, and $W$, rooted at $w$ to complete this $\Delta$-distinguishing coloring of $T$.

Color the vertices in $U \cup W$ at levels 2 through $l$ as before using only colors $1,2,3, \ldots, \Delta$. Since each such vertex at levels 2 through $l-1$ has $\Delta-1$ children, the set of colors that are used on the children of each parent is determined by the color assigned to the parent. Since $u$ and $w$ are the only level 1 vertices with the same color, we need only consider automorphisms of $T$ which interchange $u$ and $w$ or which fix $u$ and $w$.

First consider automorphisms that preserve colors and fix $u$ and $w$. From Lemma 3.2, the subtrees induced by $U$ and $W$ have $\Delta$-distinguishing colorings. These together with the coloring of the rest of $T$ gives a $\Delta$-distinguishing coloring of $T$, contradicting our hypothesis that $\chi_{D}(T)=\Delta+1$. Hence there is no nontrivial automorphism which fixes $u$ and $w$ and preserves colors.

It remains to consider automorphisms that preserve colors and interchange $u$ and $w$. Such automorphisms must interchange each vertex on level $i, 3 \leq i \leq l$, in $U$ with a uniquely determined vertex on level $i$, in $W$, that has the same sequence of colors in a path back to vertex $v$. This uniquely defines vertex $u_{1}$ in $W$ as the vertex which could be interchanged with $x$.

\section{Case 1: $x$ has at least one child.}

Without loss of generality, assume vertex $x$ has color 1 . Use color 2 on one of $x$ 's children, and color the remaining children (if any) using distinct colors from $\{3,4, \ldots, \Delta-$ 1 \}. If $u_{1}$ has $\Delta-1$ children, then no automorphism can interchange $x$ and $u_{1}$ because $x$ was chosen to have fewer than $\Delta-1$ children. Otherwise, if $u_{1}$ has at most $\Delta-2$ children, then color these children using distinct colors from $\{3,4, \ldots, \Delta\}$, and this ensures that no color-preserving automorphism can interchange $x$ and $u_{1}$. Finish the coloring of $T$ by coloring each set of siblings differently and distinctly from their parent.

\section{Case 2: $x$ has no children.}

Since $l<h$, there exists some vertex $u_{2}$ at level $l$ in $W$ which has a child. If $u_{2} \neq u_{1}$, recolor the vertices in $W$ so that the path from $w$ to $u_{2}$ has the same sequence of colors in the path from $u$ to $x$, and the other vertices in $W$ are colored so that siblings are colored differently and distinctly from their parent. With this coloring, $u_{2}$ is the only vertex in $W$ which could be interchanged with $x$ by a color-preserving automorphism. However, no automorphism can interchange $x$ with $u_{2}$ because $u_{2}$ has children and $x$ does not. As before, finish the coloring of $T$ by coloring each set of siblings differently and distinctly 
from their parent.

By the coloring we have chosen in each case, there is no automorphism that interchanges $u$ and $w$ and preserves colors. This gives a $\Delta$-distinguishing coloring of $T$, contradicting our hypothesis that $\chi_{D}(T)=\Delta+1$.

We have just shown that for trees $T, \chi_{D}(T) \leq \Delta(T)+1$, with equality precisely when $T=T_{\Delta, h}$. The next Brooks' Theorem analog shows a similar result for proper distinguishing colorings.

Theorem 3.5 For any tree, $D(T) \leq \Delta$. Furthermore, equality is achieved iff $T=T_{\Delta, h}$ for some $h \geq 0$.

Proof Cheng and Tymoczko $[3,7]$ have independently shown that if $T$ is a tree with maximum degree $\Delta \geq 2$, then $D(T) \leq \Delta$. Thus, we need only show that $D\left(T_{\Delta, h}\right) \geq \Delta$, and that if $T \neq T_{\Delta, h}$, then $D(T) \leq \Delta-1$. For the first statement, suppose that there is a distinguishing coloring of $T_{\Delta, h}$ with $\Delta-1$ colors. If $h=1$, then every child of the root must be colored distinctly, and there are $\Delta$ such children, hence we reach a contradiction. If $h \geq 2$, then consider the leaves of $T_{\Delta, h}$, which are in sets of siblings of size $\Delta-1$. If any two leaves that are siblings get the same color, there is a color-preserving automorphism of $T_{\Delta, h}$ that interchanges them and fixes all other vertices. Thus the leaves in each sibling set must receive different colors. There are $\Delta-1$ siblings and $\Delta-1$ colors, so all colors are used on each set of leaf siblings. Consider the siblings on level $h-1$. If any two siblings are colored the same, their leaf children each have the same set of colors, so there is a natural correspondence between them. Thus every set of siblings on level $h-1$ must also be colored distinctly, and use all $\Delta-1$ colors. Proceeding by induction up the levels of $T_{\Delta, h}$, we see that set of children of the root must also be colored distinctly, but there are $\Delta$ such children, hence we reach a contradiction of the fact that there is a distinguishing $\Delta-1$ labeling.

Secondly, suppose that $T$ is not $T_{\Delta, h}$ for any $\Delta, h$. We will show that $D(T) \leq \Delta-1$.

Case 1: The center of $T$ is an edge, $x y$.

Color $x$ with color 1 and $y$ with color 2. For the subtrees rooted at $x$ and $y$, color each set of siblings in $T$ distinctly. Since $x$ and $y$ are adjacent, every vertex in each subtree has at most $\Delta-1$ children, hence we can do this with $\Delta-1$ colors. By Lemma 3.2, this is a $(\Delta-1)$-distinguishing labeling.

Case 2: The center of $T$ is a vertex $v$.

If the degree of $v$ is strictly less than $\Delta$, color each set of siblings in $T$ distinctly. By Lemma 3.2, this is a $(\Delta-1)$-distinguishing coloring. Otherwise, the degree of $v$ is $\Delta$. Consider the subtrees of $T$ rooted at the children of $v$. If there are two non-isomorphic subtrees, color the corresponding children of $v$ the same, and all the other children of $v$ distinctly, using $\Delta-1$ colors, and color the rest of the tree so that the siblings sets are colored distinctly. Then no automorphism can interchange the children of $v$ which are colored the same, so they are fixed by every automorphism. By the same argument as in Lemma 3.2 , this is a $(\Delta-1)$-distinguighing labeling. 
If all subtrees rooted at the children of $v$ are isomorphic, color the leftmost $\Delta-2$ children of $v$ distinctly from the set $\{1,2,3, \ldots, \Delta-2\}$ and the last two children, say $x, y$, both with color $\Delta-1$. Color all other sibling sets distinctly. Then there is only one possible non-trivial automorphism $\sigma$ which preserves the colors. This automorphism is an involution which interchanges the subtrees rooted at $x$ and $y$, in a unique way, and fixes the subtrees rooted at the other children of $v$. Let $z$ be a vertex with shortest distance $j$ to $v$ such that the degree of $z$ is less than $\Delta$, and $z$ is contained in the subtree rooted at $y$. Each of $z$ and $\sigma(z)$ have less than or equal to $\Delta-2$ children, and each set of children must be colored distinctly. If any such $z$ has at least one child, since there are $\Delta-1$ choices of colors, we change the labeling (if necessary) so that the children of $z$ receive a different set of colors than the children of $\sigma(z)$ in the subtree rooted at $x$. This is then a $(\Delta-1)$-distinguishing labeling. If all $z$ on level $j$ in the subtree rooted at $y$, then, since all subtrees rooted at the children of $v$ are isomorphic, all vertices at level $j$ have no children and every vertex on levels 0 through $j-1$ has degree $\Delta$. Hence $T=T_{\Delta, j}$.

\section{Connected Graphs}

Theorems 3.4 and 3.5 are Brooks' Theorem type bounds for $\chi_{D}(T)$ and $D(T)$ where $T$ is a tree. In this section, we prove Brooks' theorem type bounds for $D(G)$ (Theorem 4.2) and for $\chi_{D}(G)$ (Theorem 4.5) where $G$ is a connected graph.

The statement in the first sentence of Theorem 4.2 is due to Mike Albertson and Karen Collins in 1999 (personal communication). The theorem was independently discovered by Klavžar, Wong and Zhu [6]. Our proof yields a polynomial-time algorithm as detailed in Corollary 4.3. We begin with a lemma and let $N(x)$ denote the set of neighbors of $x$.

Lemma 4.1 Suppose $G$ is a connected graph and $v$ is a vertex of $G$ and $(T, v)$ is a breadthfirst search spanning tree rooted at $v$. Let $\phi$ be a coloring (resp. proper coloring) of $V(G)$ so that the vertices at distance at most $i$ from $v$ in $T$ are fixed by every automorphism of $G$ which preserves colors, and the vertices at distance greater than $i$ from $v$ in $T$ are colored so that each vertex is colored differently from its siblings in $T$. Then $\phi$ is a distinguishing labeling (resp. proper distinguishing labeling).

Proof Let $\sigma$ be an automorphism of $G$ which preserves colors. Consider the vertices of $G$ in the order they were selected in forming $T$, namely $v, v_{1}, v_{2}, \ldots, v_{n}$. Since $i \geq 0$, $\sigma(v)=v$. By induction, we may assume $v, v_{1}, v_{2}, \ldots, v_{j}$ are fixed by $\sigma$ and consider $v_{j+1}$ at distance greater than $i$ from $v$ in $T$. Its parent, $x$, is fixed, so $\sigma\left(v_{j+1}\right) \in N(x)$. We know $\sigma\left(v_{j+1}\right)$ is not a sibling of $v_{j+1}$ in $T$ because $\sigma$ is color-preserving and these siblings are colored differently. By construction, all other vertices in $N(x)$ are in the set $v, v_{1}, v_{2}, \ldots, v_{j}$ and hence are already fixed by $\sigma$. Thus $\sigma\left(v_{j+1}\right)=v_{j+1}$.

Theorem 4.2 If $G$ is a connected graph with maximum degree $\Delta$, then $D(G) \leq \Delta+1$. Furthermore, equality is achieved if and only if $G=K_{\Delta+1}, K_{\Delta, \Delta}$ or $C_{5}$. 
Proof By Theorem 2.2, $D(G)=\Delta+1$ for $G=K_{\Delta+1}, K_{\Delta, \Delta}$ and $C_{5}$, thus it remains to show $D(G) \leq \Delta$ for all other graphs $G$. The only connected graph with $\Delta=1$ is $K_{2}$, and the connected graphs with $\Delta=2$ are paths and cycles, all of whose distinguishing numbers are in Table 1. For the rest of the argument, we assume that $\Delta \geq 3$. Let $G$ be a connected graph with $\Delta \geq 3$ other than $K_{\Delta+1}$ or $K_{\Delta, \Delta}$.

Case 1: $G$ has a vertex $v$ with degree less than $\Delta$.

Let $T$ be a breadth-first search spanning tree of $G$ with $v$ as its root. Color $v$ with color $\Delta$ and retire the color $\Delta$. Color the remaining vertices so that each vertex is colored differently from its siblings in $T$. This is possible since each sibling set has at most $\Delta-1$ vertices. By Lemma 4.1 with $i=0$, this coloring is $\Delta$-distinguishing.

Case 2: $\quad G$ is regular of degree $\Delta$ and there is a triple of vertices $v, w, y$ where $v w, v y \in E(G)$ and $N(w)-y=N(y)-w$.

First suppose $N(x)-y=N(y)-x$ for all vertices $x$ with $x v \in E(G)$. If $w y \in E(G)$ then $G=K_{\Delta+1}$ and if $w y \notin E(G)$ then $G=K_{\Delta, \Delta}$, each yielding a contradiction.

Otherwise, since $\Delta \geq 3$, there exists a vertex $x \neq w, y$ such that $v x \in E(G)$, but $N(x)-y \neq N(y)-x$. Rearrange $T$ as necessary to let $w$ be chosen as the first child of $v$, and $x, y$ chosen as the last two children of $v$. Color $v$ with color $\Delta$ and retire the color $\Delta$. Color the leftmost $\Delta-2$ neighbors of $v$ distinctly from the set $\{1,2,3, \ldots,(\Delta-2)\}$, so $w$ has color 1 , and use color $\Delta-1$ for both $x, y$. Thus, $w$ and $y$ have distinct colors. Proceed as in Case 1 to extend the coloring to the descendants of neighbors of $v$, coloring the children of each vertex distinctly. We show this coloring is $\Delta$-distinguishing.

Vertex $v$ is pinned, and since the leftmost $\Delta-2$ children of $v$ in $T$ are labeled distinctly, they must also be pinned. Each descendant in $T$ of these children is also pinned. If $x, y$ were also pinned, in spite of being labeled with the same color, then the coloring is $\Delta$ distinguishing. So, we need only consider an automorphism, say $\sigma$, which interchanges $x$ and $y$. Since $N(x)-y \neq N(y)-x$ and $G$ is $\Delta$-regular, there exists a vertex $u \neq x$ with $u y \in E(G)$ but $u x \notin E(G)$. Thus $u=w$ or $u \in N(y)-w=N(w)-y$ and so $u$ is either one of the leftmost $\Delta-2$ children of $v$ in $T$ or a child of $w$ in $T$. In either instance, $u$ is pinned and we have $\sigma(u)=u$. However, $\sigma(y)=x$ so $u y \in E(G)$ implies $\sigma(u) \sigma(y)=u x \in E(G)$, a contradiction.

Case 3: $G$ is regular of degree $\Delta$ and $N(x)-y \neq N(y)-x$ for every triple of vertices $v, x, y$ where $v x, v y \in E(G)$.

Choose any vertex $v$, and let $T$ be a breadth-first search spanning tree with $v$ as its root. Color $v$ with color $\Delta$ and retire the color $\Delta$. Label the leftmost $\Delta-2$ neighbors of $v$ distinctly from the set $\{1,2,3, \ldots,(\Delta-2)\}$, and the last two neighbors, say $x, y$, both with $\Delta-1$. Then $v$ is pinned, and since the leftmost $\Delta-2$ neighbors of $v$ are labeled distinctly, they must also be pinned. Proceed as in Case 1 to extend the labeling to the rest of the graph, coloring the children in $T$ of each vertex distinctly, except for $y$ and its descendants in $T$. If there is no non-trivial automorphism which interchanges $x$ and $y$, we extend the labeling by coloring the descendants in $T$ of $y$ so that each vertex is colored distinctly from its siblings. By Lemma 4.1, this is a $\Delta$-distinguishing labeling.

Otherwise, $v$ its leftmost $\Delta-2$ children and all their descendants in $T$ are pinned, and any non-trivial automorphism, $\sigma$, that preserves the colors must interchange $x$ and 
$y$. Let $S_{x}$ be the set of children of $x$ in $T$ that are not neighbors of $y$ and let $S_{y}$ be the set of children of $y$ in $T$ (which will not be neighbors of $x$ because $x$ comes before $y$ in the breadth-firstsearch spanning tree $T$ ). Since $\sigma$ interchanges $x$ and $y$ it must also interchange $S_{x}$ with $S_{y}$.

We first show that $\left|S_{y}\right|>0$. Since $N(y)-x \neq N(x)-y$, and $G$ is regular of degree $\Delta$, there must be some vertex $z \neq x$ which is adjacent to $y$ but not $x$. Then $z$ cannot be any vertex already pinned because $\sigma$ interchanges $x$ and $y$. Thus, $z$ must be a child of $x$ or $y$ in $T$ and since $z x \notin E(G)$ we have $z \in S_{y}$. This means that $\left|S_{y}\right|>0$.

Subcase 3a: $1 \leq\left|S_{y}\right| \leq \Delta-2$.

We distinguish $y$ from $x$ by using a different set of colors on $S_{y}$ than are used on $S_{x}$, but still coloring the vertices in $S_{y}$ distinctly. We can do this since there are $\left(\begin{array}{c}\Delta-1 \\ \left|S_{y}\right|\end{array}\right) \geq 2$ possible sets of colors for the children of $y$. This pins $x$ and $y$. We extend the labeling by coloring the descendants of vertices in $S_{y}$ in the same fashion. By Lemma 4.1 with $i=1$, this is a $\Delta$-distinguishing labeling.

Subcase 3b: $\left|S_{y}\right|=\Delta-1$.

In this subcase $x$ and $y$ have no common neighbors. We distinguish $x$ from $y$ by using a different set of colors on $S_{y}$ than are used on $S_{x}$, but coloring the rightmost two vertices of $S_{y}$ the same. Let these vertices be $u_{1}, u_{2}$. So we color the leftmost $\Delta-3$ vertices of $S_{y}$ distinctly from the set $\{1,2,3, \ldots, \Delta-3\}$ and color both $u_{1}, u_{2}$ with color $\Delta-2$. This pins $x$ and $y$, and all vertices in the graph except $u_{1}$ and $u_{2}$ and their descendants in $T$.

Thus, we need only consider automorphisms $\sigma$ which interchange $u_{1}$ and $u_{2}$. By the stipulations of Case 3 (with $v=y, x=u_{1}$ and $y=u_{2}$ ) we know $N\left(u_{1}\right)-u_{2} \neq N\left(u_{2}\right)-u_{1}$. However, $G$ is regular of degree $\Delta$, so there exists a vertex $z \neq u_{1}$ that is adjacent to $u_{2}$ but not $u_{1}$. If vertex $z$ is already fixed by $\sigma$, then this fixes $u_{2}$ and our coloring is a $\Delta$-distinguishing labeling of $G$ as desired. Otherwise, $z$ is a child of $u_{2}$ in $T$.

If $u_{2}$ has between 1 and $\Delta-2$ children in $T$, we proceed as in Subcase 3a; if it has exactly $\Delta-1$ children in $T$, we proceed to the next level of $T$. By induction on the number of levels of $T$, this process must eventually halt, giving us a $\Delta$-distinguishing labeling of $G$.

Corollary 4.3 Our proof of Theorem 4.2 provides a polynomial-time algorithm to find a $\Delta$-distinguishing labeling of a connected graph $G$ (provided $G \neq K_{\Delta+1}, K_{\Delta, \Delta}$ or $C_{5}$ ).

Proof Input a graph $G$ with maximum degree $\Delta$. Each of the steps described below can be accomplished in polynomial time. First check that $G \neq K_{\Delta}, K_{\Delta, \Delta}$. If $\Delta=1,2, G$ is a path or a cycle, and we distinguish as in Section 1. Otherwise, $\Delta \geq 3$. Find the degree of each vertex in $G$. If there a vertex of degree less than $\Delta$, use the coloring described in Case 1. Otherwise, $G$ is regular of degree $\Delta$ and we select a vertex $v$ arbitrarily and construct a breadth-first search spanning tree $T$ with $v$ as the root. For each pair $x, y$ of children of $v$, check whether $N(x)-y=N(y)-x$. If such a pair $x, y$ is found, then use the coloring in Case 2.

Otherwise color as in Case 3 , that is, color $v$ with $\Delta$ and retire $\Delta$. Label the leftmost $\Delta-2$ neighbors of $v$ distinctly from the set $\{1,2,3, \ldots,(\Delta-2)\}$, and the last two neighbors, say $x, y$, both with $\Delta-1$. Proceed as in Case 1 to extend the labeling to the 
rest of the graph, coloring the children in $T$ of each vertex distinctly, except for $y$ and its descendants in $T$. Compute the set $S_{y}$, which will be non-empty. If $\left|S_{y}\right| \leq \Delta-2$, finish the coloring as in Subcase 3a. Otherwise, $\left|S_{y}\right|=\Delta-1$. We finish the coloring of $G$ by coloring the descendants of $y$ as follows. Consider these vertices in the order that they were selected in $T$, coloring siblings distinctly, unless the parent is $y$ or a rightmost descendant of $y$ with $\Delta-1$ children in $T$. For $y$ and each rightmost descendant of $y$ with $\Delta-1$ children in $T$, color its children with $1,2,3, \ldots, \Delta-3, \Delta-2, \Delta-2$. When all of $T$ has been colored, then all vertices are pinned except possibly for the rightmost two children (and their descendants in $T$ ) of the last, rightmost descendant of $y$ with $\Delta-1$ children in $T$, say $w$, with rightmost two children $u_{1}, u_{2}$. If $N\left(u_{1}\right)=N\left(u_{2}\right)$, we re-label $G$, starting with $w$ as the root, and proceeding as in Case 2. Since the initial coloring is done in polynomial time, doing it a second time is still polynomial time. If $N\left(u_{1}\right) \neq N\left(u_{2}\right)$, then $u_{2}$ has at least one child in $T$, but $u_{2}$ does not have $\Delta-1$ children in $T$, since $w$ was the last rightmost descendant of $y$ to do so. Thus we recolor the children of $u_{2}$ as in Case 3a with $x=u_{1}$ and $y=u_{2}$. This yields a $\Delta$-distinguishing labeling of $G$ in polynomial time.

In Theorem 4.2, the hypothesis that $G$ is a connected graph is necessary. For example, consider the graph $G$ consisting of two copies of $K_{3}$. Graph $G$ is regular of degree 2, but $D(G)=4$, since we cannot label the two triangles with identical sets of labels. This example can be generalized; to get a graph which is regular of degree 2 with distinguishing number $s+1$, take $\left(\begin{array}{l}s \\ 3\end{array}\right)+1$ copies of $K_{3}$. However, we do have the following theorem, which does not require $G$ to be connected and generalizes one direction of Theorem 2.4.

Theorem 4.4 Let $H_{1}, H_{2}, \ldots, H_{t}$ be pairwise non-isomorphic connected graphs and let $G$ be the disjoint union of $\alpha_{i}$ copies of $H_{i}$ for $1 \leq i \leq t$. Then $D(G) \leq p$ where $p$ is the smallest integer for which $\left(\begin{array}{c}p \\ D\left(H_{i}\right)\end{array}\right) \geq \alpha_{i}$ for all $1 \leq i \leq t$.

Proof For each $i$, we must find a different distinguishing coloring of each copy of $H_{i}$. Since $\left(\begin{array}{c}p \\ D\left(H_{i}\right)\end{array}\right) \geq \alpha_{i}$, there are at least $\alpha_{i}$ different sets of colors we can use to distinguish each $H_{i}$ differently.

For some graphs $H_{i}$, it is possible to find more than one different distinguishing labeling using the same set of colors. For instance, $D\left(K_{3,3}\right)=4$, using two different sets of 3 different colors on each independent set. There are 4 different sets of 3 different colors from $\{1,2,3,4\}$, hence there are $\left(\begin{array}{l}4 \\ 2\end{array}\right)=6$ different ways to distinguish $K_{3,3}$ with 4 colors. Hence the bound in Theorem 4.4 is not tight in general.

Theorem 4.5 For all connected graphs $G, \chi_{D}(G) \leq 2 \Delta(G)$. Furthermore, equality is achieved if and only if $G=K_{\Delta, \Delta}$ or $C_{6}$.

Proof By Theorem 2.2, $\chi_{D}(G)=2 \Delta(G)$ for $G=K_{\Delta, \Delta}$ and $C_{6}$. Thus it remains to show $\chi_{D}(G) \leq 2 \Delta(G)-1$ for all other graphs. As noted before, the only connected graph with $\Delta=1$ is $K_{2}$, and the connected graphs with $\Delta=2$ are paths and cycles, all of whose 
distinguishing chromatic numbers are in Table 1. For the rest of the argument, we assume that $\Delta \geq 3$.

Let $G$ be a connected graph with $\Delta \geq 3$ other than $G=K_{\Delta, \Delta}$ or $C_{6}$. In each case below we will choose a vertex $v \in V(G)$ and let $T$ be a breadth-first search spanning tree of $G$ with $v$ as the root. By the definition of a breadth-first search spanning tree, the distance between $v$ and any vertex $w$ in $T$ (its level) is the same as the distance between $v$ and $w$ in $G$.

Case 1: $G$ has a vertex $v$ of degree less than $\Delta$.

We color $G$ as follows. Color $v$ with color $2 \Delta-1$, and then retire color $2 \Delta-1$. This pins $v$. We color the rest of $G$ using colors from the set $\{1,2,3, \ldots, 2 \Delta-2\}$. Consider the vertices at distance $i$ from $v$, and color the vertices from the left to the right, in the order they were chosen to be in $T$, so that each vertex receives the first color that does not appear among its (at most) $\Delta-2$ siblings and its (at most) $\Delta-1$ neighbors which have been already colored. Since $\Delta-2+\Delta-1=2 \Delta-3$, such a greedy coloring is possible. It is a proper distinguishing labeling by Lemma 4.1 .

Case 2: $\quad G$ is regular of degree $\Delta$ and there is a triple of vertices $v, w, y$ where $v w, v y \in E(G)$ and $N(w)-y=N(y)-w$.

First suppose $N(x)-y=N(y)-x$ for all vertices $x$ with $x v \in E(G)$. If $w y \in E(G)$ then $G=K_{\Delta+1}$ which has $\chi_{D}(G)=\Delta+1 \leq 2 \Delta$ as desired. If $w y \notin E(G)$ then $G=K_{\Delta, \Delta}$, a contradiction.

Otherwise, there exists a vertex $x \neq w, y$ such that $v x \in E(G)$, but $N(x)-y \neq$ $N(y)-x$. Rearrange $T$ as necessary to let $w$ be chosen as the first child of $v$, and $x, y$ chosen as the last two children of $v$. We use the same greedy coloring algorithm as in Case 1 , but could run into difficulty at level 1 , where there are $\Delta$ children of $v$, hence $y$, has $\Delta-1$ siblings and $\Delta-1$ neighbors already colored from the set $\{1,2,3, \ldots, 2 \Delta-2\}$. There is a color for $y$ unless the set of siblings of $y$ is distinct from the set of neighbors of $y$, and all $2 \Delta-2$ vertices are colored distinctly. In this instance, all neighbors of $y$ are vertices on level 2 of $T$ and in particular, $x y \notin E(G)$. Thus $N(x)=N(x)-y$ and $N(y)=N(y)-x$ and thus $N(x) \neq N(y)$.

Now we may color $y$ the same color as $x$. Then any nontrivial automorphism of $G$ that preserves the colors must interchange $x$ and $y$ and fix all other children of $v$ in $T$. Since $w$ comes first in $T$, each vertex in $N(y)$ is either $w$ itself or a child of $w$ in $T$, and thus the vertices in $N(y)$ are fixed by any automorphism that preserves the colors. Since $N(x) \neq N(y)$, there is no automorphism which both preserves the colors and interchanges $y$ and $x$. Thus we have a proper $(2 \Delta-1)$-distinguishing labeling of $G$ as desired.

Case 3: $G$ is regular of degree $\Delta$ and $N(x)-y \neq N(y)-x$ for every triple of vertices $v, x, y$ where $v x, v y \in E(G)$.

Choose any vertex $v$, and let $T$ be a breadth-first search spanning tree with $v$ as its root. Color the graph as in Case 2, from the bottom to the top, left to right, where each vertex is colored distinctly from its neighbors and siblings, except that, if necessary, we color the rightmost two children of $v$, say $x$ and $y$, both the same color. If $x$ and $y$ get different colors then this is a proper $(2 \Delta-1)$-distinguishing labeling of $G$ and we are done. Otherwise, as before, $y$ 's set of siblings is disjoint from its set of neighbors. In particular, 
$y x \notin E(G)$ and so $N(x)=N(x)-y$ and $N(y)=N(y)-x$.

Then $v$, its leftmost $\Delta-2$ children and all their descendants in $T$ are pinned, and any non-trivial automorphism, $\sigma$, that preserves the colors must interchange $x$ and $y$. Let $S_{x}$ be the set of children of $x$ in $T$ that are not neighbors of $y$ and let $S_{y}$ be the set of children of $y$ in $T$ (which are not neighbors of $x$ because $x$ comes before $y$ in the breadth-first search spanning tree $T$ ). Since $\sigma$ interchanges $x$ and $y$ it must also interchange $S_{x}$ with $S_{y}$.

We first show that $\left|S_{y}\right|>0$. Since $N(y) \neq N(x)$, and $G$ is regular of degree $\Delta$, there must be some vertex $z$ which is adjacent to $y$ but not $x$. Then $z$ cannot be any vertex already pinned because $\sigma$ interchanges $x$ and $y$. Thus, $z$ must be a child of $x$ or $y$ in $T$ and since $z x \notin E(G)$ we have $z \in S_{y}$. This means that $\left|S_{y}\right|>0$.

Subcase 3a: $1 \leq\left|S_{y}\right| \leq \Delta-2$.

Let $u$ be the rightmost child of $y$ in $T$. Note that $u$ is not adjacent to any vertex on level 1 except for $y$, because $T$ is a breadth-first search spanning tree. In the initial coloring, $u$ is colored distinctly from all of its up to $\Delta-1$ colored neighbors and at most $\Delta-2$ siblings. Thus, the color of $u$ is not forced-there is at least one alternate choice of color for $u$. Changing the color of $u$ will not affect the coloring of any level 1 vertex except for $y$, and will not change the color of any vertex on a level greater than 1 , because $u$ is the last vertex to be colored before the level 1 vertices. If it is possible to change the color of $u$ to the same color as the vertex $x$ (say red), we do so; then there is another color that we can color $y$ which is different from all of its siblings and all of its neighbors and we have a proper $(2 \Delta-1)$-distinguishing labeling of $G$ as desired.

If this is not possible, then $u$ must have a neighbor (besides $y$ ) that is red. This means that there is an alternate color for $u$ which is different from red. Change $u$ 's color to this alternate color and leave $y$ colored red. Since the vertices of $S_{y}$ are colored distinctly as are the vertices in $S_{x}, \sigma$ only preserves the colors if the set of colors in $S_{y}$ is the same as the set of colors in $S_{x}$. If this is true in the initial coloring, it will not be true when we change the color of $u$. Thus, the set of colors on the neighbors of $y$ will be different from the set of colors on the neighbors of $x$, so each of them will be pinned by the coloring, and this will be a proper $(2 \Delta-1)$-distinguishing labeling.

Subcase 3b: $\left|S_{y}\right|=\Delta-1$.

In this subcase, $N(y)=\{v\} \cup S_{y}$, so $x$ and $y$ have no common neighbors other than $v$. If the set of colors in $S_{x}$ is different from the set of colors in $S_{y}$, color $y$ the same color as $x$. Any $\sigma$ which preserves the colors must fix $x$ and $y$, hence fixing the whole graph. Otherwise we assume that the $(\Delta-1)$ colors in $S_{x}$ are identical to the $(\Delta-1)$ colors in $S_{y}$, and that these colors, together with the $(\Delta-1)$ colors on the children of $v$ partition the set of colors $\{1,2,3, \ldots, 2 \Delta-2\}$. Let $u_{1}, u_{2}$ be the two rightmost children of $y$ in $T$. We colored $u_{2}$ last of the vertices on level 2. If $u_{1} u_{2} \in E(G)$ then the set of siblings of $u_{2}$ intersects its neighbor set so it is possible to change the color of $u_{2}$.

If not, then $u_{1} u_{2} \notin E(G)$ we change the color of $u_{2}$ to be the same as the color of $u_{1}$. Then, any non-trivial $\sigma$ which preserves the colors must fix $x$ and $y$, because now their neighbor sets of colors are different, and interchange $u_{1}$ and $u_{2}$. Such a $\sigma$ must also fix all vertices in $G$ except for descendants of $u_{1}$ and $u_{2}$. By the stipulations of Case 3 (with $v=y, x=u_{1}$ and $\left.y=u_{2}\right)$ and the fact that $u_{1} u_{2} \notin E(G)$, we know $N\left(u_{1}\right) \neq N\left(u_{2}\right)$. 
However, $G$ is regular of degree $\Delta$, so there exists a vertex $z$ that is adjacent to $u_{2}$ but not $u_{1}$. If vertex $z$ is already fixed by $\sigma$, then this fixes $u_{2}$ and our coloring is a $2 \Delta-1$ distinguishing labeling of $G$ as desired. Otherwise, $z$ is a child of $u_{2}$ in $T$.

If $u_{2}$ has between 1 and $\Delta-2$ children in $T$, we proceed as in Subcase 3a; if it has exactly $\Delta-1$ children in $T$, we proceed to the next level of $T$. By induction on the number of levels of $T$, this process must eventually halt, giving us a $2 \Delta-1$ distinguishing labeling of $G$.

This proof also results in a polynomial time algorithm for $(2 \Delta-1)$-proper distinguishing of a graph. The proof is similar to that of Corollary 4.3, hence is omitted.

Corollary 4.6 Let connected $G \neq K_{n, n}$, with $\Delta(G) \geq 3$. The proof of Theorem 4.5 gives a polynomial time algorithm to find a proper distinguishing $(2 \Delta-1)$-labeling of $G$.

The hypothesis of connected is once again necessary. A graph $G$ consisting of multiple copies of $K_{3,3}$ has $\Delta(G)=3$. Each copy requires six colors by Theorem 2.3, three for each partite set. Distinct sets of three colors must be used for each partite set in each copy. To get a graph which is regular of degree 3 with distinguishing chromatic number $s+1$, take $\frac{1}{2}\left(\begin{array}{l}s \\ 3\end{array}\right)+1$ copies of $K_{3,3}$. For instance, if $s=6$, then $\left(\begin{array}{l}6 \\ 3\end{array}\right)=20$, so six colors suffice for the graph connsisting of 10 copies of $K_{3,3}$, but the graph consisting of 11 copies of $K_{3,3}$ has $\chi_{D}=7$.

We do not know of a graph for which $\chi_{D}=2 \Delta-1$, but we have a construction which shows there are infinitely many graphs for which $\chi_{D}=2 \Delta-2$. Let $H$ be the graph which is $K_{n, n}$ plus two new vertices $u$, $v$, with $u$ adjacent to one independent set of $K_{n, n}$ and $v$ adjacent to the other. Note that the vertices in the $K_{n, n}$ in $H$ must all be colored distinctly in a proper distinguishing labeling of $H$. For any graph $G$ of maximum degree $n+1$, replace every edge of $G$ by a copy of $H$, that is, for every edge $\{x, y\}$, add a copy of $H$, say $H_{x y}$ and let $x$ be adjacent to $u_{x y}$ and $y$ be adjacent to $v_{x y}$, and remove the edge between $x$ and $y$. Then the new graph has maximum degree $n+1$, and every copy of $H$ requires at least $2 n$ colors to distinguish.

Corollary 4.7 Let $G \neq K_{\Delta, \Delta}$ be a connected graph with root $w$ and $\Delta(G) \geq 3$. Then $\chi_{D}(G, w) \leq 2 \Delta(G)-2$.

Proof Choose the root $w$ as the initial vertex in the proof of Theorem 4.5, then proceed with the rest of the proof. Since $w$ has at most $\Delta$ neighbors, and there are $2 \Delta-2$ colors available, we can choose a color for $w$ to make a proper coloring, which must also be distinguishing since every automorphism of $(G, w)$ must fix $w$.

Although choosing a root for $G$ reduces the distinguishing number for graphs with maximum degree at least 3 , the situation for $\Delta=2$ is different. Note that $\chi_{D}\left(C_{2 k+1}, w\right)=$ $\chi_{D}\left(C_{2 k+1}\right)=3$ is not reduced by choosing a root, since 3 colors are needed for a proper coloring of an odd cycle. Also, $\chi_{D}\left(C_{2 k}, w\right)=3$, because any two coloring of an even cycle that fixes one vertex (the root) has a reflection about the fixed vertex and its opposite 


\begin{tabular}{|l|c|c|}
\hline & Conn. graphs w/max. & Upper bounds for others \\
\hline$\chi$ & $K_{\Delta+1}, C_{2 k+1}$ & $\Delta-1$ \\
\hline$D$ & $K_{\Delta+1}, K_{\Delta, \Delta}, C_{5}$ & $\Delta-1$ \\
\hline$\chi_{D}$ & $K_{\Delta, \Delta}, C_{6}$ & $2 \Delta-1$ or $2 \Delta-2$ \\
\hline \hline & Trees w/max. & Upper bounds for others \\
\hline$\chi$ & & 2 \\
\hline$D$ & $T_{\Delta, h}$ & $\Delta-1$ \\
\hline$\chi_{D}$ & $T_{\Delta, h}$ & $\Delta$ \\
\hline
\end{tabular}

Table 2: Graphs with maximum values in terms of $\Delta$, and upper bounds for all other graphs

vertex on the even cycle. The distinguishing chromatic number of a cycle is only reduced by choosing a root in the case of $C_{4}$ and $C_{6}$.

A summary of Brooks' Theorem type results appears in Table 4.

\section{Conjectures}

As described in Section $4, K_{n, n}$ and $C_{6}$ satisfy $\chi_{D}(G)=2 \Delta$, and there are graphs where $\chi_{D}(G)=2 \Delta-2$. Every other connected graph has $\chi_{D}(G) \leq 2 \Delta-1$, but there are no known cases of equality. In our proof, we used the color $2 \Delta-1$ exactly once. It may be that all graphs which are not $K_{n, n}$ or $C_{6}$ have a proper $(2 \Delta-2)$-distinguishing labeling which uses every color at least twice.

Conjecture 5.1 There is no connected graph $G$ such that $\chi_{D}(G)=2 \Delta-1$.

The examples of graphs where $\chi_{D}(G)=2 \Delta-2$ depend heavily on complete bipartite subgraphs for their high chromatic distinguishing number. Thus, we conjecture:

Conjecture 5.2 Let the girth of connected graph $G$ be 5 or greater. Then $\chi_{D}(G) \leq \Delta+1$.

The authors would like to thank the referee for many helpful suggestions.

\section{References}

[1] M. O. Albertson and K. L. Collins, Symmetry Breaking in Graphs, Electron. J. of Combin., 3 (1996) \#R18.

[2] Brooks' Theorem, see for example: Bondy and Murty, Graph Theory with Applications, North Holland, 1976.

[3] C. T. Cheng, Three problems in graph labeling, Ph. D. dissertation, The Johns Hopkins University, 1999. 
[4] R. L. Graham, M. Grötschel, L. Lovász, editors, Handbook of Combinatorics, Vols. I and II, The MIT Press, Cambridge MA, 1995.

[5] J. Gross and J. Yellen, editors, Handbook of Graph Theory, CRC Press, Boca Raton, 2004.

[6] S. Klavzar, T. Wong and X. Zhu, Distinguishing labelings of group action on vector spaces and graphs, preprint.

[7] J. Tymoczko, Distinguishing numbers for graphs and groups, Electronic Journal of Combinatorics 11 (1) 2004, \#R63. (Also available at arXiv:math.CO/0406542.) 